\title{
Nonlinear operation of resonant sensors based on weakly- coupled resonators: theory and modeling
}

\author{
Jérôme Juillard, Ali Mostafa, Pietro Maris Ferreira
}

\begin{abstract}
This theoretical work investigates the properties of nonlinearlyoperated weakly-coupled resonators (WCRs) for resonant sensing applications. We propose an analysis framework for mutually injection-locked oscillators (MILOs) and mode-localized oscillators (MOLOs), subject to nonlinear restoring and damping forces. Under some simplifying assumptions, three sensor architectures are investigated and compared, highlighting several common features such as: (i) the insensitivity of the amplitude ratio output metric to the Af effect, (ii) the instability of one oscillation state above a threshold amplitude. These results are illustrated and validated using transient simulations. Their range of validity is then discussed with respect to finite perturbations, finite bandwidth, measurement noise and nonlinear dissipation-fluctuation.
\end{abstract}

\section{Introduction}

The last few years have seen a growing interest in resonant MEMS sensors based on weakly-coupled resonators (WCRs). Such sensors consist in two or more resonators [14], that are coupled in such a way that a small change of one mechanical parameter (typically the stiffness or the mass of one resonator) induces a large change of an electrically-measurable output metric. Another interesting property of such sensors is their robustness to drift, since their typical output metrics are intrinsically differential, as illustrated in [4-5].

As opposed to frequency-modulated (FM) "conventional" resonant sensors based on a single oscillator [6], WCRs are usually amplitude-modulated (AM) or phase-modulated (PM). In "mode-localized" sensors (MOLOs), the ratio of the oscillation amplitudes of (two of) the resonators is the output metric of choice [1-2, 5], but other amplitude-based metrics have also been proposed [7-8]. In "mutually injection-locked oscillators" (MILOs) based on two synchronized oscillators, the phase-difference between the two oscillating structures is usually preferred as output metric [3-4], although it was recently shown that, in some MILO architectures, amplitude ratio could also be used [9].

Our theoretical analyses $[3,10]$ have highlighted that the large parametric sensitivity of PM or AM WCRs is commensurate with their sensitivity to thermomechanical noise. This restatement of the classical result [11] that coupling $N$ noisy resonators may at most yield a $\sqrt{N}$ improvement in signal-to-noise ratio was experimentally verified on a PM MILO in [12]. Even though, a large parametric sensitivity is very interesting for resonators that are limited by measurement noise rather than by intrinsic noise sources [13]. Another item of interest is the performance of WCRs in the nonlinear oscillation regime, to which this paper is dedicated. 
While nonlinear operation of WCRs has been experimentally investigated in [9, 14-15], and nonlinear coupling of FM resonant sensors or oscillators was covered in [16], there is little theoretical background against which experimental results obtained with nonlinear WCRs can be tested. Our own work on this subject $[9,17]$ has highlighted that some MILOs may benefit from nonlinear operation. In particular, the increase of the measurement range of MILOs, with a trade-off in sensitivity, was theoretically and experimentally proven in [9]. Most interestingly, the analysis in [17] showed the resolution of a MILO based on two similar Duffing resonators may not be limited by the A-f effect [18] provided amplitude-ratio was used as an output metric instead of phasedifference. To this day, no similar study has been performed on MOLOs. Furthermore, the results in [17] are limited to a narrow framework: a specific MILO architecture, only nonlinear restoring forces and quasi-static perturbations being considered.

With this paper, we establish the fundamental limitations of WCRs in the nonlinear regime, aiming at a general, qualitative description of their behavior, rather than at particular results. The analysis of WCRs presented in section II may be used for MILOs or MOLOs; it is slightly simplified compared to the one in [17], but is more general in the sense that it captures dynamic system fluctuations (i.e. finite sensor bandwidth). Furthermore, it is not only valid for resonators with nonlinear restoring forces (e.g stress-stiffening, or electrostatic softening [19]), but also with nonlinear damping forces. Nonlinear damping is ubiquitous in MEMS sensors: for instance, it may result from the squeezed-film phenomenon [20], from anchor loss [21], or from coupling to an undesired vibration mode [22]. A global outlook on the nonlinear properties of WCRs with quadratic stiffness and damping coefficients is derived in sections III and IV. In section III, three WCR architectures are investigated, and their properties are established with further simplifying assumptions. We find that the insensitivity of AM WCRs to the A-f effect holds in all the studied cases. In section IV, these results are illustrated and compared to system-level simulations. Our simplifying hypotheses are also discussed and commented, and the impact of finite, dynamic perturbations is investigated. Section V is dedicated to concluding remarks and perspectives. These results are experimentally investigated in a separate paper [23].

\section{General framework and model}

We consider a system consisting of two coupled resonators with nominally equal natural frequencies. One seeks to measure the relative stiffness mismatch of the resonators through the phase-difference or the amplitude-ratio of the detected motional signals. Such a system can be described by the following set of non-dimensional equations:

$$
\begin{aligned}
& \left(1+\epsilon+\gamma_{x} x^{2}\right) x+\left(\frac{1}{Q_{x}}+\alpha_{x} x^{2}\right) \frac{d x}{d t}+\frac{d^{2} x}{d t^{2}}=f_{x}\left(x, \frac{d x}{d t}, y, \frac{d y}{d t}\right)+n_{x}(t) \\
& \left(1-\epsilon+\gamma_{y} y^{2}\right) y+\left(\frac{1}{Q_{y}}+\alpha_{y} y^{2}\right) \frac{d y}{d t}+\frac{d^{2} y}{d t^{2}}=f_{y}\left(x, \frac{d x}{d t}, y, \frac{d y}{d t}\right)+n_{y}(t)
\end{aligned}
$$


where $t$ is time, $x$ and $y$ are the motional signals, $f_{x}$ and $f_{y}$ are the forces used for driving the resonators and coupling them, $n_{x}$ and $n_{y}$ are independent random forces typically resulting from thermomechanical fluctuations, $Q_{x}$ and $Q_{y}$ are the resonators' quality factors, $\gamma_{x}$ and $\gamma_{y}$ are nonlinear stiffness coefficients, $\alpha_{x, y} \geq 0$ are nonlinear damping coefficients and $\epsilon$ is (half) the relative stiffness mismatch one seeks to measure. The stiffness of the first (resp. second) resonator is an increasing (resp. decreasing) function of $\epsilon$, and so is its resonance frequency. Consequently, the average resonance frequency of the resonators is independent of $\epsilon$. A generic cubic restoring force (or Duffing model) is assumed: $\gamma_{x, y}>0$ may adequately describe dominant stress-stiffening effects, while $\gamma_{x, y}<0$ describes moderate electrostatic softening effects [19]. The cubic damping force model which we assume is also rather ubiquitous [24], and corresponds quite accurately to the experimental results presented in [23].

Equation (1) can be studied by averaging [25] or harmonic balance techniques [26]. For example, a slowly-varying amplitude and phase model can be established as follows, with the same approach as in [3]. We first assume that

$x(t)=X(t) \sin \left(\Omega t+\theta_{x}(t)\right), \quad y(t)=Y(t) \sin \left(\Omega t+\theta_{y}(t)\right)$

where $X(t), Y(t), \theta_{x}(t)$ and $\theta_{y}(t)$ are assumed to vary on a time-scale much slower than the steady-state period $2 \pi / \Omega$ of the system. Projecting (1-a) on $\sin \left(\Omega t+\theta_{x}\right)$, $\cos \left(\Omega t+\theta_{x}\right)$, and (1-b) on $\sin \left(\Omega t+\theta_{y}\right)$ and $\cos \left(\Omega t+\theta_{y}\right)$ yields:

$\ddot{X}+\left(\frac{1}{Q_{x}}+\frac{1}{4} \alpha_{x} X^{2}\right) \dot{X}+X\left(1+\epsilon+\frac{3}{4} \gamma_{x} X^{2}-\Omega_{x}^{2}\right)=f_{\sin x}+n_{\sin x}(3-\mathrm{a})$

$X \dot{\Omega}_{x}+2 \dot{X} \Omega_{x}+\left(\frac{1}{Q_{x}}+\frac{1}{4} \alpha_{x} X^{2}\right) X \Omega_{x}=f_{\cos x}+n_{\cos x}$

$\ddot{Y}+\left(\frac{1}{Q_{y}}+\frac{1}{4} \alpha_{y} Y^{2}\right) \dot{Y}+Y\left(1-\epsilon+\frac{3}{4} \gamma_{y} Y^{2}-\Omega_{y}^{2}\right)=f_{\text {siny }}+n_{\text {siny }}(3-\mathrm{c})$

$Y \dot{\Omega}_{y}+2 \dot{Y} \Omega_{y}+\left(\frac{1}{Q_{y}}+\frac{1}{4} \alpha_{y} Y^{2}\right) Y \Omega_{y}=f_{\text {cos } y}+n_{\text {cos } y}$

where a dot denotes differentiation with respect to time, $\Omega_{x}=\Omega+\dot{\theta}_{x}$ and $\Omega_{y}=\Omega+\dot{\theta}_{y}$. In the rest of the paper, we refer to the terms appearing on the right-hand side of (3-a) or (3-c) as "in phase" terms, whereas those appearing on the right-hand side of (3-b) or (3-d) are "quadrature" terms. As our previous analyses have shown, the first term on the left-hand side of quadrature equations and the first 2 terms on the left-hand side of the in-phase equations have little influence on the slow dynamics of amplitude and phase and can be neglected [3]. By assumption, $\dot{\theta}_{x}, \dot{\theta}_{y} \ll \Omega$, the quadrature equations become:

$2 \dot{X} \Omega+\left(\frac{1}{Q_{x}}+\frac{1}{4} \alpha_{x} X^{2}\right) X \Omega=f_{\cos x}+n_{\cos x}$ 
$2 \dot{Y} \Omega+\left(\frac{1}{Q_{y}}+\frac{1}{4} \alpha_{y} Y^{2}\right) Y \Omega=f_{\cos y}+n_{\cos y}$

and the in-phase equations can be re-arranged to yield:

$\Omega \dot{\phi}=-\left(\epsilon+\frac{3}{8} \gamma_{x} X^{2}-\frac{3}{8} \gamma_{y} Y^{2}\right)+\frac{1}{2 X}\left(f_{\sin x}+n_{\sin x}\right)-\frac{1}{2 Y}\left(f_{\sin y}+n_{\sin y}\right)$

where $\phi=\theta_{y}-\theta_{x}$, and

$\Omega^{2}=1+\frac{3}{8} \gamma_{x} X^{2}+\frac{3}{8} \gamma_{y} Y^{2}-\frac{1}{2 X}\left(f_{\sin x}+n_{\sin x}\right)-\frac{1}{2 Y}\left(f_{\sin y}+n_{\sin y}\right)$.

This leads to:

$\dot{X}=-\left(\frac{1}{Q_{x}}+\frac{1}{4} \alpha_{x} X^{2}\right) \frac{X}{2}+\frac{1}{2 \Omega}\left(f_{\cos x}+n_{\cos x}\right)$

$\dot{Y}=-\left(\frac{1}{Q_{y}}+\frac{1}{4} \alpha_{y} Y^{2}\right) \frac{Y}{2}+\frac{1}{2 \Omega}\left(f_{\cos y}+n_{\cos y}\right)$

$\dot{\phi}=-\frac{1}{\Omega}\left(\epsilon+\frac{3}{8} \gamma_{x} X^{2}-\frac{3}{8} \gamma_{y} Y^{2}\right)+\frac{1}{2 \Omega X}\left(f_{\sin x}+n_{\sin x}\right)-\frac{1}{2 \Omega Y}\left(f_{\sin y}+n_{\sin y}\right)$

Note that no particular assumption concerning the nature of the coupling is made in deriving (7), so that this model may be used to study indifferently MILOs or MOLOs.

For a given value of $\epsilon$, the steady state of the system, $\boldsymbol{s}_{\epsilon}=\left(X_{\epsilon}, Y_{\epsilon}, \phi_{\epsilon}\right)^{T}$ and its steadystate angular frequency $\Omega_{\epsilon}$ can then be determined by setting to zero the left-hand sides of (7) and the noise sources appearing on the right-hand sides, and solving the algebraic equations resulting from (6-7). Except in trivial cases, these usually have no analytical solutions, and must be solved numerically. The stability of a steady-state solution, or its sensitivity to a given system parameter (typically $\epsilon$ in a resonant sensing application) can then be determined by letting $X=X_{\epsilon}+\delta X, Y=Y_{\epsilon}+\delta Y$ and $\phi=\phi_{\epsilon}+\delta \phi$ in (7), and studying the resulting linearized system. This is similar to the approach presented in [3], except that we make the hypothesis that $\Omega$ does not deviate from its steady-state value $\Omega_{\epsilon}$, which simplifies our problem with little loss of accuracy, as shown in section III.

We may formally rewrite (7) as

$\frac{d \boldsymbol{s}}{d t}=\boldsymbol{F}(\boldsymbol{s}, \boldsymbol{p})$,

where $\boldsymbol{p}$ are fluctuating system parameters or parameters to which one seeks to determine the sensitivity of the system's state. For any perturbation $\delta \boldsymbol{p}$, we find the corresponding perturbation of the system's state satisfies:

$\left(\frac{d}{d t}-J_{s}\right) \delta s=J_{p} \delta p$

where $\boldsymbol{J}_{\boldsymbol{s}}$ is the jacobian of $\boldsymbol{F}$ with respect to state $\boldsymbol{s}$, and $\boldsymbol{J}_{\boldsymbol{p}}$ is the jacobian of $\boldsymbol{F}$ with respect to parameters $\boldsymbol{p}$, evaluated at the steady-state. If only quasistatic parameter 
fluctuations are considered, as may be deemed sufficient for a rough sensitivity analysis, the time derivatives may be neglected in such a formulation, as in $[10,17]$. The stability of the steady state may also be trivially determined from the perturbed set of equations (9). This approach may be generalized to an arbitrary set of coupled resonators with little difficulty.

Along with an observation equation, linking the system's state to an output metric $M$ :

$M=g(\boldsymbol{s}, \boldsymbol{q}),(10)$

where $\boldsymbol{q}$ is a set of parameters (possibly distinct from $\boldsymbol{p}$ ), equation (9) can be used to derive the transfer function between any of the system's parameters and any output metric as:

$\delta M=\frac{\partial g}{\partial s}\left(\frac{d}{d t}-\boldsymbol{J}_{s}\right)^{-1} \boldsymbol{J}_{\boldsymbol{p}} \delta \boldsymbol{p}+\frac{\partial g}{\partial \boldsymbol{q}} \delta \boldsymbol{q}$

This generic formulation is well-suited for parametric sensitivity, noise sensitivity and bandwidth analysis of resonant sensors based on WCRs. For example, it may readily be used as the basis of a simulation tool for deriving numerical values of such quantities, for many self-oscillating systems based on WCRs, such as MILOs or the MOLOs discussed in [13-15]. However, such numerical results, lacking in physical insight, do not make the properties of WCRs much easier to grasp. This is why, in the following section, we give a more in-depth look at some particular systems, hoping to achieve a more intuitive understanding of WCRs in the nonlinear regime.

Two MILO architectures, for which both the phase difference $\phi$ and the amplitude ratio $R=X / Y$ can be used as output metrics, are studied in sub-section III-A. The case of closed-loop mode-localized sensors, for which the amplitude ratio is the output metric of choice, is also briefly treated in sub-section III-B. These results are illustrated and commented in section IV.

\section{Properties of WCRs in the nonlinear regime}

In this section, the output metrics of three WCRs are analyzed in terms of their sensitivity to mismatch $\epsilon$ and of their sensitivity to intrinsic noise sources (e.g. thermomechanical), modeled by $n_{x}$ and $n_{y}$ in (1). Assuming these noise sources are independent and of similar magnitude, the sensitivity to noise of $M$, an output metric, is defined as the quadratic mean of the sensitivities to each independent noise component:

$\frac{\partial M}{\partial n} \equiv \sqrt{\left(\frac{\partial M}{\partial n_{\sin x}}\right)^{2}+\left(\frac{\partial M}{\partial n_{\sin y}}\right)^{2}+\left(\frac{\partial M}{\partial n_{\cos x}}\right)^{2}+\left(\frac{\partial M}{\partial n_{\cos y}}\right)^{2}}$.

Within the system's bandwidth, this quantity sets the noise floor of the system. As in [17], we define the figure of merit (FOM) of any output metric $M$ as the absolute value of the ratio of its sensitivity to mismatch $\epsilon$ over its sensitivity to intrinsic noise: 
$\mathrm{FOM}_{\text {int }}(M) \equiv\left|\frac{\partial M}{\partial \epsilon} / \frac{\partial M}{\partial n}\right|$

The larger this figure of merit is, the better the output metric performs with respect to intrinsic noise sources, which ultimately limit the performance of conventional frequency-modulated resonant sensors [27]. In this section, we focus on the derivation of this FOM close to the nominal value $\epsilon=0$, assuming (i) quasistatic fluctuations of $\epsilon$ and of quadrature and in-phase noise components, (ii) no measurement noise. The limits of these assumptions are discussed and commented in section IV.

\section{III-A Mutually injection-locked oscillators}

Consider the MILO depicted in Fig. 1. Because of the digital nature of the mixer, the excitation forces (at the mixer outputs) are binary-valued, and independent of the motional signal amplitudes. Their duty cycles (which sum to 50\%) depend on $\phi$, and consequently so do their quadrature and in-phase components. Furthermore, these also depend on $\theta=\theta_{a c t}+\theta_{\text {comp }}+\theta_{\text {det }}$, the delay (expressed in radians) in each feedback loop, as follows [3]:

$$
\begin{array}{ll}
f_{\sin x}=\frac{F_{x}}{\pi}(\cos \theta+\cos (\theta+\phi)) & f_{\cos x}=\frac{F_{x}}{\pi}(\sin \theta+\sin (\theta+\phi)) \\
f_{\sin y}=\frac{F_{y}}{\pi}(\cos \theta-\cos (\theta-\phi)) & f_{\cos y}=\frac{F_{y}}{\pi}(\sin \theta-\sin (\theta-\phi))
\end{array}
$$

where $F_{x}$ and $F_{y}$ are the peak values of the binary excitation signals of the resonators. Note that, in this theoretical study, $\theta$ is a convenient parameter which sums up the actual delays and phase-shifts occurring in the different blocks of the feedback paths [4].

Accounting for infinitesimal fluctuations (denoted by a $\delta$ ) of mismatch $\epsilon$, and driving force amplitudes $F_{x}$ and $F_{y}$, the linearized dynamics of the MILO's state (9) can be written explicitly as:

$$
\begin{aligned}
& \frac{\dot{\delta} X}{X_{\epsilon}}=-\frac{1}{2}\left(\frac{1}{Q_{x}}+\frac{3}{4} \alpha_{x} X_{\epsilon}^{2}\right) \frac{\delta X}{X_{\epsilon}}+\frac{f_{\cos x}^{\prime}}{2 \Omega_{\epsilon} X_{\epsilon}} \delta \phi+\frac{1}{2 \Omega_{\epsilon} X_{\epsilon}}\left(n_{\cos x}+\frac{\delta F_{x}}{F_{x}} f_{\cos x}\right) \\
& \frac{\dot{\delta} Y}{Y_{\epsilon}}=-\frac{1}{2}\left(\frac{1}{Q_{y}}+\frac{3}{4} \alpha_{y} Y_{\epsilon}^{2}\right) \frac{\delta Y}{X_{\epsilon}}+\frac{f_{\cos y}^{\prime}}{2 \Omega_{\epsilon} Y_{\epsilon}} \delta \phi+\frac{1}{2 \Omega_{\epsilon} Y_{\epsilon}}\left(n_{\cos y}+\frac{\delta F_{y}}{F_{y}} f_{\cos y}\right)
\end{aligned}
$$

$\dot{\delta \phi}=-\left(\frac{3}{4} \gamma_{x} X_{\epsilon}^{2}+\frac{f_{\operatorname{sinx}}}{2 X_{\epsilon}}\right) \frac{\delta X}{\Omega_{\epsilon} X_{\epsilon}}+\left(\frac{3}{4} \gamma_{y} Y_{\epsilon}^{2}+\frac{f_{\operatorname{siny}}}{2 Y_{\epsilon}}\right) \frac{\delta Y}{\Omega_{\epsilon} Y_{\epsilon}}+\left(\frac{f_{\text {sinx }}^{\prime}}{2 \Omega_{\epsilon} X_{\epsilon}}-\frac{f_{\text {siny }}^{\prime}}{2 \Omega_{\epsilon} Y_{\epsilon}}\right) \delta \phi-\frac{\delta \epsilon}{\Omega_{\epsilon}}+$

$\frac{1}{2 \Omega_{\epsilon} X_{\epsilon}}\left(n_{\sin x}+\frac{\delta F_{x}}{F_{x}} f_{\sin x}\right)-\frac{1}{2 \Omega_{\epsilon} Y_{\epsilon}}\left(n_{\sin y}+\frac{\delta F_{y}}{F_{y}} f_{\sin y}\right)$

where the prime denotes a differentiation with respect to $\phi$.

In what follows, these expressions are simplified under the assumption that the resonators are nominally identical $\left(Q_{x, y}=Q, \alpha_{x, y}=\alpha, \gamma_{x, y}=\gamma, F_{x, y}=F, \epsilon=0\right)$. Furthermore, driving forces fluctuations (i.e. $\delta F_{x}=\delta F_{y}=0$ ) are neglected. Including 
these terms in (15) is however useful to understand how the sensitivity to noise of a MILO may be studied indirectly through its sensitivity to drive level, as we do in [23].

\section{III-A-1 MILO architecture with $\theta=90^{\circ}$}

Choosing $\theta=90^{\circ}$ leads to two possible synchronized oscillation states, with $\phi_{0}= \pm 90^{\circ}$ and $X_{0}=Y_{0}=A[3,28]$ (hence $R_{0}=1$ ). The steady-state oscillation amplitude satisfies:

$A\left(\frac{1}{Q}+\frac{1}{4} \alpha A^{2}\right)=\frac{1}{\Omega_{0}} \frac{F}{\pi}$

so that, for small values of the driving force we have $A \propto F$, whereas for larger values $A \propto F^{1 / 3}$. From (6), and supposing $\left|\Omega_{0}-1\right| \ll 1$, we also have

$\Omega_{0} \approx 1+\frac{3}{8} \gamma A^{2} \pm \frac{1}{2}\left(\frac{1}{Q}+\frac{1}{4} \alpha A^{2}\right)$

Using (16) and (17), the perturbed dynamics (15) at $\phi_{0}= \pm 90^{\circ}$ can be rewritten:

$\frac{\dot{\delta} X}{A}=-\frac{1}{2}\left(\frac{1}{Q}+\frac{3}{4} \alpha A^{2}\right) \frac{\delta X}{A} \mp \frac{1}{2}\left(\frac{1}{Q}+\frac{1}{4} \alpha A^{2}\right) \delta \phi+\frac{n_{\cos x}}{2 \Omega_{0} A}$

$\frac{\dot{\delta} Y}{A}=-\frac{1}{2}\left(\frac{1}{Q}+\frac{3}{4} \alpha A^{2}\right) \frac{\delta Y}{A} \pm \frac{1}{2}\left(\frac{1}{Q}+\frac{1}{4} \alpha A^{2}\right) \delta \phi+\frac{n_{\cos y}}{2 \Omega_{0} A}$

$\dot{\delta \phi}=-\left(\frac{3}{4 \Omega_{0}} \gamma A^{2} \mp \frac{1}{2}\left(\frac{1}{Q}+\frac{1}{4} \alpha A^{2}\right)\right)\left(\frac{\delta X}{A}-\frac{\delta Y}{A}\right)-\frac{\delta \epsilon}{\Omega_{0}}+\frac{n_{\sin x}}{2 \Omega_{0} A}-\frac{n_{\sin y}}{2 \Omega_{0} A}$.

When the time-derivative terms on the left-hand side of (18) are dropped, a set of equations governing the quasi-static fluctuations (with noise and/or mismatch) of $X, Y$ and $\phi$ is obtained. These quasi-static equations are valid when considering perturbations below the cutoff frequency of the system (which is trivially found to be $1 / 2 Q$ when all nonlinearities are neglected). From (18), it is then readily found that:

$\delta R=\frac{\delta X}{A}-\frac{\delta Y}{A}=\frac{\partial R}{\partial \epsilon} \times\left(\delta \epsilon-\frac{n_{\sin x}}{2 A}+\frac{n_{\sin y}}{2 A}\right)$,

$\frac{\delta \phi}{\phi_{0}}=\frac{1}{\phi_{0}} \frac{\partial \phi}{\partial \epsilon} \times\left(\delta \epsilon-\frac{n_{\sin x}}{2 A}+\frac{n_{\sin y}}{2 A}\right)+\frac{1}{F}\left(n_{\cos x}-n_{\cos y}\right)$

where

$$
\begin{gathered}
\frac{\partial R}{\partial \epsilon}= \pm \frac{1}{\Omega_{0}} \times \frac{2}{\frac{1}{Q}+\left(\frac{1}{4} \alpha \mp \frac{3}{2 \Omega_{0}} \gamma\right) A^{2}} \\
\frac{1}{\phi_{0}} \frac{\partial \phi}{\partial \epsilon}=-\frac{1}{\pi} \frac{\frac{1}{Q}+\frac{3}{4} \alpha A^{2}}{\frac{1}{Q}+\frac{1}{4} \alpha A^{2}} \times \frac{\partial R}{\partial \epsilon}
\end{gathered}
$$

From (12) and (18), the sensitivities to noise can be expressed as

$\frac{\partial R}{\partial n}=\frac{\sqrt{2}}{2 A}\left|\frac{\partial R}{\partial \epsilon}\right|$, 

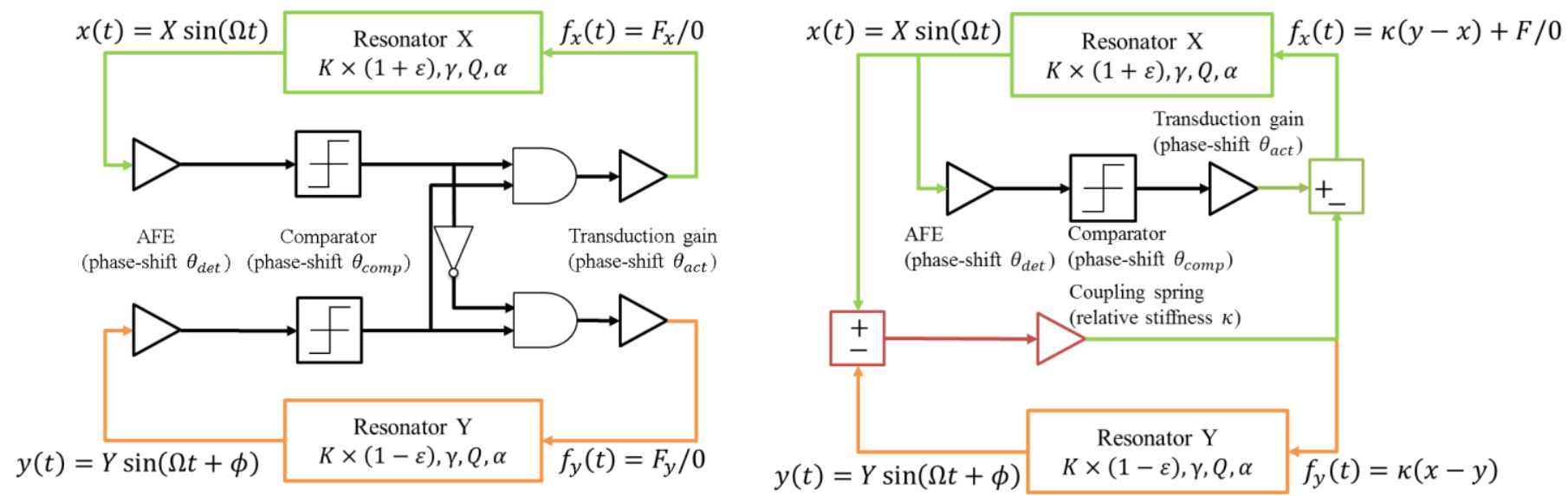

Fig. 1. System-level view of a MILO based on a digital Fig. 2. System-level view of a mode-localized oscillator, as in mixer, as in [4].

[14][29].

$\left|\frac{1}{\phi_{0}}\right| \frac{\partial \phi}{\partial n}=\frac{1}{A} \times\left|\frac{1}{\phi_{0}} \frac{\partial \phi}{\partial \epsilon}\right| \times \sqrt{\frac{1}{2}+\frac{1}{2}\left(\frac{\frac{1}{Q}+\frac{3}{2}\left(\frac{\alpha}{4} \mp \frac{\gamma}{\Omega_{0}}\right) A^{2}}{\frac{1}{Q}+\frac{3}{4} \alpha A^{2}}\right)^{2}}$

Setting $\gamma=0$ and $\alpha=0$ in (21) shows that, in the linear regime, the FOM (13) of the amplitude ratio is $\sqrt{2}$ larger than that of the phase difference: this can be understood with (19), which makes plain that $R$ is only affected by in-phase noise $\left(n_{\sin x}, n_{\sin y}\right)$, as opposed to $\phi$ which is also affected by quadrature noise $\left(n_{\cos x}, n_{\cos y}\right)$. The FOM of both output metrics increases linearly with oscillation amplitude $A$.

The situation becomes dramatically different in the nonlinear regime. Let us first consider the case when $\alpha=0$ and $\gamma \neq 0$. First of all, it is clear that only one of the two synchronized states is stable at large oscillation amplitudes: assume, for example, that $\gamma>0$ and $\phi_{0}=90^{\circ}$, and, for the sake of simplicity, that $\Omega_{0}=1$. Then, as shown by (20), the sensitivity to mismatch, and hence to noise (21) or to any other perturbation becomes infinite as $A$ reaches the critical amplitude

$A_{\text {Duff }}=\sqrt{\frac{2}{3|\gamma| Q}}$

and the system becomes unstable. On the other hand, still assuming that $\gamma>0$, the equilibrium corresponding to $\phi_{0}=-90^{\circ}$ is stable regardless of the oscillation amplitude ${ }^{1}$. In this case, when $A \gg A_{D u f f}$, the sensitivity to mismatch (20) of both $R$ and $\phi$ decreases as $1 / A^{2}$; as illustrated in [9], this decrease of sensitivity is accompanied by an increase of the MILO's locking range. Furthermore, as shown by (21), the sensitivity to noise of $R$ then decreases as $1 / A^{3}$, whereas that of $\phi$ decreases as $1 / A$, because of the A-f coupling term appearing in the square root on the right-hand side. Hence, the FOM of the amplitude ratio still increases linearly with $A$, as it does below $A_{D u f f}$, whereas that of

1 The situation is reversed when $\gamma<0$, i.e. the equilibrium corresponding to $\phi_{0}=-90^{\circ}$ is unstable above the critical amplitude, whereas the one corresponding to $\phi_{0}=90^{\circ}$ is stable. 
the phase difference starts decreasing as $1 / A$ at this point. In other words, phase difference measurements are limited by the A-f effect, whereas amplitude ratio measurements are not. Note that, from (17), the condition that $\left|\Omega_{0}-1\right| \ll 1$ is equivalent to $A \ll \sqrt{Q} \times A_{D u f f}$.

When $\alpha>0$ and $\gamma=0$, the oscillation amplitude increases as $F^{1 / 3}$ above the critical damping amplitude

$A_{d a m p}=\frac{2}{\sqrt{\alpha Q}}$

as (16) shows. Thus, when nonlinear damping is present, the FOM of $R$ still increases as $A$, but increasing it by an order of magnitude requires increasing the driving force by three orders of magnitude (consequently, oscillation amplitudes much larger than $A_{\text {damp }}$ are not likely to be encountered in practice). Furthermore, the FOM of $\phi$ also increases as $A$, as the rightmost term in (21) tends to a constant when $A>A_{\text {damp }}$. Thus, the FOM of both output metrics may be improved by increasing the drive level. However, as discussed in section IV, the proposed FOM may then lose its significance due to nonlinear dissipation-fluctuation. Note that both steady-state solutions $\phi_{0}= \pm 90^{\circ}$ remain stable in that case. Letting $\Omega_{0}=1$ in $(20-a)$, we find that the two solutions are in fact stable provided $\alpha>6|\gamma|$ (since this implies the denominator of the right-hand side of $(20-\mathrm{a})$ is positive irrespective of $A)$.

The case when $\alpha$ and $|\gamma|$ are commensurate may also open interesting avenues of research. In particular, when $\alpha=6|\gamma|$, then the equilibrium corresponding to $\phi_{0}=90^{\circ}$ in the case $\gamma>0$, or to $\phi_{0}=-90^{\circ}$ in the case $\gamma<0$, has some remarkable properties: as can be seen by letting $\Omega_{0}=1$ in $(20-\mathrm{a})$, the sensitivity of $R$ to $\epsilon$ becomes completely independent of oscillation amplitude. This does not change the FOM of this output metric, but is likely quite interesting in the case when measurement noise dominates, as discussed in section IV. Still in the case $\alpha=6|\gamma|$, the sensitivity of $\phi$ to $\epsilon$ is not constant, as (20-b) shows, but is an increasing function of $A$, which levels out above $A_{\text {damp }}$ to 3 times its value in the linear regime. However, matching in practice the nonlinear damping and stiffness coefficients, by clever design or by tuning, might prove quite a challenge, depending on the technology used for the resonators. Furthermore, this result relies on the assumption that $\Omega_{0}$ is independent of amplitude, which is not actually the case (17), so that even if $\alpha=6|\gamma|$, the parametric sensitivity of both output metrics decreases beyond a certain threshold. This is illustrated in section II-C.

It should also be noted that the linear increase of the FOM of $R$ with the oscillation amplitude $A$ is independent of the degree of the nonlinear restoring or damping forces, as can be readily shown, whereas the behavior of the FOM of $\phi$ is nonlinearitydependent. 
III-A-2 MILO with $\theta=45^{\circ}$ or $\theta=135^{\circ}$

When $\theta=45^{\circ}$ or $135^{\circ}$, only one oscillation state is possible instead of two, even in the linear regime [3]. In the nominal state of the system, for $\epsilon=0$, we find $X_{0}=Y_{0}=A$ (hence $R_{0}=1$ ), and $\phi_{0}= \pm 90^{\circ}$ when $\theta=90^{\circ} \mp 45^{\circ}$. The steady-state amplitude satisfies

$A\left(\frac{1}{Q}+\frac{1}{4} \alpha A^{2}\right)=\sqrt{2} \times \frac{F}{\Omega \pi}$

and the angular frequency is

$\Omega_{0} \approx 1+\frac{3}{8} \gamma A^{2}$.

The linearized dynamics can be written:

$\frac{\delta X}{A}=-\frac{1}{2}\left(\frac{1}{Q}+\frac{3}{4} \alpha A^{2}\right) \frac{\delta X}{A} \mp \frac{1}{4}\left(\frac{1}{Q}+\frac{1}{4} \alpha A^{2}\right) \delta \phi+\frac{n_{\cos x}}{2 \Omega_{0} A}$

$\frac{\delta Y}{A}=-\frac{1}{2}\left(\frac{1}{Q}+\frac{3}{4} \alpha A^{2}\right) \frac{\delta Y}{A} \pm \frac{1}{4}\left(\frac{1}{Q}+\frac{1}{4} \alpha A^{2}\right) \delta \phi+\frac{n_{\cos y}}{2 \Omega_{0} A}$

$\dot{\delta \phi}=-\frac{1}{\Omega_{0}} \frac{3}{4} \gamma A^{2}\left(\frac{\delta X}{A}-\frac{\delta Y}{A}\right)-\frac{1}{2}\left(\frac{1}{Q}+\frac{1}{4} \alpha A^{2}\right) \delta \phi-\frac{\delta \epsilon}{\Omega_{0}}+\frac{n_{\sin x}}{2 \Omega_{0} A}-\frac{n_{\sin y}}{2 \Omega_{0} A}$

As far as quasistatic perturbations are concerned, we find:

$\delta R=\frac{\partial R}{\partial \epsilon} \times\left(\delta \epsilon-\frac{n_{\sin x}}{2 A}+\frac{n_{\sin y}}{2 A} \pm\left(\frac{n_{\cos x}}{2 A}-\frac{n_{\cos y}}{2 A}\right)\right)$,

$\frac{\delta \phi}{\phi_{0}}=\frac{1}{\phi_{0}} \frac{\partial \phi}{\partial \epsilon} \times\left(\delta \epsilon-\frac{n_{\sin x}}{2 A}+\frac{n_{\operatorname{siny}}}{2 A} \pm\left(\frac{n_{\cos x}}{2 A}-\frac{n_{\cos y}}{2 A}\right)\right)+\frac{\sqrt{2}}{F}\left(n_{\cos x}-n_{\cos y}\right)$.

The sensitivities to mismatch:

$\frac{\partial R}{\partial \epsilon}= \pm \frac{1}{\Omega_{0}} \times \frac{2}{\frac{1}{Q}+\left(\frac{3}{4} \alpha \mp \frac{3}{2 \Omega_{0}} \gamma\right) A^{2}}$

$\frac{1}{\phi_{0}} \frac{\partial \phi}{\partial \epsilon}=-\frac{2}{\pi} \frac{\frac{1}{Q}+\frac{3}{4} \alpha A^{2}}{\frac{1}{Q}+\frac{1}{4} \alpha A^{2}} \times \frac{\partial R}{\partial \epsilon}$.

and to noise

$\frac{\partial R}{\partial n}=\frac{1}{A}\left|\frac{\partial R}{\partial \epsilon}\right|$,

$\left|\frac{1}{\phi_{0}}\right| \frac{\partial \phi}{\partial n}=\frac{\sqrt{2}}{2 A} \times\left|\frac{1}{\phi_{0}} \frac{\partial \phi}{\partial \epsilon}\right| \times \sqrt{1+\left(\frac{\frac{3}{2 \Omega_{0}} \gamma A^{2}}{\frac{1}{Q}+\frac{3}{4} \alpha A^{2}}\right)^{2}}$.

are trivially derived from (27)-(28). From (29), we find that the system is stable above $A_{\text {Duff }}$ provided either of these conditions is met: 
- $\gamma>0$ and $\theta=135^{\circ}$ (corresponding to $\phi_{0}=-90^{\circ}$ ).

- $\gamma<0$ and $\theta=45^{\circ}$ (corresponding to $\phi_{0}=90^{\circ}$ ).

Otherwise, the system becomes unstable once $A_{D u f f}$ is reached (if $\alpha=0$ ), or, more generally, when the denominator on the right-hand side of (29-a) is cancelled out (which occurs if $\alpha<2|\gamma|)$.

Note that, in the linear regime, the situation is reversed compared to the $\theta=90^{\circ}$ case, i.e. phase difference is a slightly better output metric than amplitude ratio, because the quadrature noise terms then cancel out in (28). However, in the presence of nonlinear restoring forces, the same behavior as in the $\theta=90^{\circ}$ case is observed: the FOM of the phase difference output metric is limited by the A-f effect, whereas that of the amplitude ratio is not. In the presence of dominant nonlinear damping forces, the same trends as in the $\theta=90^{\circ}$ case are also observed, i.e. the FOM of both output metrics keep increasing linearly with $A$, with $A \propto F^{1 / 3}(30)$.

Finally, letting $\Omega_{0}=1$ in (29-a), we find that the threshold point $\alpha=2|\gamma|$ is of particular interest: when $\gamma>0$ (resp. $\gamma<0$ ), the sensitivity to $\epsilon$ of the $\theta=45^{\circ}$ (resp. $135^{\circ}$ ) MILO becomes nearly independent of oscillation amplitude, as far as $R$ is concerned, or increases and levels out above $A_{d a m p}$, as far as $\phi$ is concerned. As previously, this has little impact on the FOM of either output metric, but may be interesting in the case of dominant measurement noise.

\section{III-B Mode-localized oscillator}

Consider the closed-loop mode-localized oscillator in Fig. 2, whose architecture is similar to the one in [14][29]. For this system, the driving and coupling terms appearing in (7) are

$f_{\sin x}=-\kappa X+\kappa Y \cos \phi, f_{\cos x}=\frac{F}{\pi}-\kappa Y \sin \phi$

$f_{\text {siny }}=-\kappa Y+\kappa X \cos \phi, f_{\text {cosy }}=\kappa X \sin \phi \cdot(31-\mathrm{b})$

In the absence of mismatch $\left(Q_{x, y}=Q, \alpha_{x, y}=\alpha, \gamma_{x, y}=\gamma, F_{x}=F, F_{y}=0, \epsilon=0\right)$, we find from (7) and (31), that two oscillation states are possible, with $X_{0}=Y_{0}=A$ (hence $R_{0}=1$ ) and

$\sin \phi_{0}=\frac{\Omega_{0}}{\kappa}\left(\frac{1}{Q}+\frac{1}{4} \alpha A^{2}\right)$

where, supposing $\sin \left|\phi_{0}\right| \ll 1$ so that $\cos \phi_{0} \approx \pm 1$, we have from (6) and (31)

$\Omega_{0} \approx 1+\frac{3}{8} \gamma A^{2}+\frac{1}{2}(\kappa \mp \kappa)$.

The linearized dynamics can be written, close to either of these equilibria: 
$\frac{\delta X}{A}=-\frac{1}{2}\left(\frac{1}{Q}+\frac{3}{4} \alpha A^{2}\right) \frac{\delta X}{A}-\frac{1}{2}\left(\frac{1}{Q}+\frac{1}{4} \alpha A^{2}\right) \frac{\delta Y}{A} \mp \frac{\kappa}{2 \Omega_{0}} \delta \phi+\frac{n_{\cos x}}{2 \Omega_{0} A}$.

$(34-a)$

$\frac{\delta Y}{A}=\frac{1}{2}\left(\frac{1}{Q}+\frac{3}{4} \alpha A^{2}\right) \frac{\delta X}{A}-\frac{1}{2}\left(\frac{1}{Q}+\frac{1}{4} \alpha A^{2}\right) \frac{\delta Y}{A} \pm \frac{\kappa}{2 \Omega_{0}} \delta \phi+\frac{n_{\cos y}}{2 \Omega_{0} A}$.

(34-b)

$\dot{\delta \phi}=-\frac{1}{\Omega_{0}}\left(\frac{3}{4} \gamma A^{2} \pm \kappa\right)\left(\frac{\delta X}{A}-\frac{\delta Y}{A}\right)-\frac{\delta \epsilon}{\Omega_{0}}+\frac{n_{\sin x}}{2 \Omega_{0} A}-\frac{n_{\sin y}}{2 \Omega_{0} A}$.

Equation (34-c) then yields the sensitivity of the amplitude ratio to quasistatic fluctuations of mismatch:

$\frac{\partial R}{\partial \epsilon}=-1 /\left(\frac{3}{4} \gamma A^{2} \pm \kappa\right)$

and to noise:

$\frac{\partial R}{\partial n}=\frac{\sqrt{2}}{2 A}\left|\frac{\partial R}{\partial \epsilon}\right|$

The same general behavior as in the previous two sub-sections can be inferred. As in the MILO case, parametric sensitivity decreases as $A$ increases above the critical amplitude:

$A_{\text {Duff }}=\sqrt{\frac{4|\kappa|}{3|\gamma|}}$

with only one of the two oscillation states being stable:

- $\quad$ the in-phase mode when $\gamma \kappa>0$.

- the anti-phase mode when $\gamma \kappa<0$.

To complete the parallel with MILOs, one should point out that this analysis holds provided $\sin \left|\phi_{0}\right| \ll 1$, which, in presence of nonlinear damping, boils down to stating that the oscillation amplitude should be smaller than

$A_{\text {damp }}=2 \sqrt{\frac{|\kappa|}{\alpha}}$

Thus, although damping does not appear explicitly in (35)-(36) it still sets a limit to the performance of MOLOs, as it does in the linear case [10].

Transient simulations of (1) clearly confirm these results. To the best of our knowledge, the experimental studies of nonlinear MOLOs available in the literature (see [30], or [31] published after the initial submission of this paper) are limited to the analysis of a single oscillation state, or of both oscillation states below the critical amplitude (37), and do not contradict them. 
Our simulations show that the sensitivity to mismatch of the considered MOLO also decreases with oscillation amplitude when operating far from the veering zone, i.e. with $|\epsilon|>|\kappa|$. As in the linear case [10], only one oscillation state is possible, depending on whether the resonance frequency of the first resonator is higher than that of the second resonator (i.e. depending on the sign of $\epsilon$ ) and on the sign of coupling factor $\kappa$.

\section{Illustrations}

The sensitivities and FOMs obtained close to $\epsilon=0$ for the coupled architectures of sections III-A and III-B are represented in Fig. 3, 4 and 5, for the following sets of parameters: $Q=1000, \gamma=-2 \times 10^{-3}$ and $\alpha=0$ (Fig. 3), $\gamma=0$ and $\alpha=5 \times 10^{-4}$ (Fig. 4), or $\gamma=-2 \times 10^{-3}$ and $\alpha=5 \times 10^{-4}$ (Fig. 5). For the case of the mode-localized architecture, the coupling constant is taken equal to $\kappa=10^{-1}$. The curves of Fig. 3-5 correspond to the unconditionally stable modes of the studied architectures, in the case $\gamma<0: \phi_{0}=90^{\circ}$ for the MILOs with $\theta=90^{\circ}$ and with $\theta=45^{\circ}$, the anti-phase mode for the MOLO.

Let us first consider Fig. 3, in which only nonlinear restoring forces are present $(\alpha=0)$. For $\gamma=-2 \times 10^{-3}$, we find $A_{D u f f} \approx 0.58$ in the case of MILOs (represented by a thin vertical line), and $A_{D u f f} \approx 8.2$ in the case of the mode-localized architecture. It is visible that:

- below $A_{D u f f}$, the sensitivity to mismatch obtained with MILOs is several orders of magnitudes larger than that of the mode-localized architecture, whereas the mode-localized architecture has the smallest sensitivity to noise. Regardless of the output metric, all sensitivities to noise decrease as $1 / A$, while sensitivities to mismatch are independent of $A$.

- above $A_{D u f f}$, the sensitivity to mismatch of all architectures decreases as $1 / A^{2}$. The sensitivity to noise of $R$ decreases as $1 / A^{3}$ for all architectures, while that of $\phi$ decreases as $1 / A$.

- consequently the FOM of $\phi$ decreases as $1 / A$ above $A_{D u f f}$, whereas that of $R$ increases as $A$, regardless of which architecture is considered.

In Fig. 4, only nonlinear damping forces are accounted for $(\gamma=0)$. For $\alpha=5 \times 10^{-4}$, we find $A_{\text {damp }} \approx 2.8$ in the case of MILOs . For the mode-localized architecture, the hypothesis that $\sin \left|\phi_{0}\right| \ll 1$ is respected below $A_{\text {damp }} \approx 25$ (note that the fact that $\sin \left|\phi_{0}\right|$ becomes finite is not accounted for in the simulations). Above $A_{d a m p}$, the force required to reach a given amplitude increases as $A^{2}$. The most striking differences with the previous case are that:

- above $A_{\text {damp }}$, the sensitivity to noise of all MILO output metrics decreases as $1 / A^{3}$. 

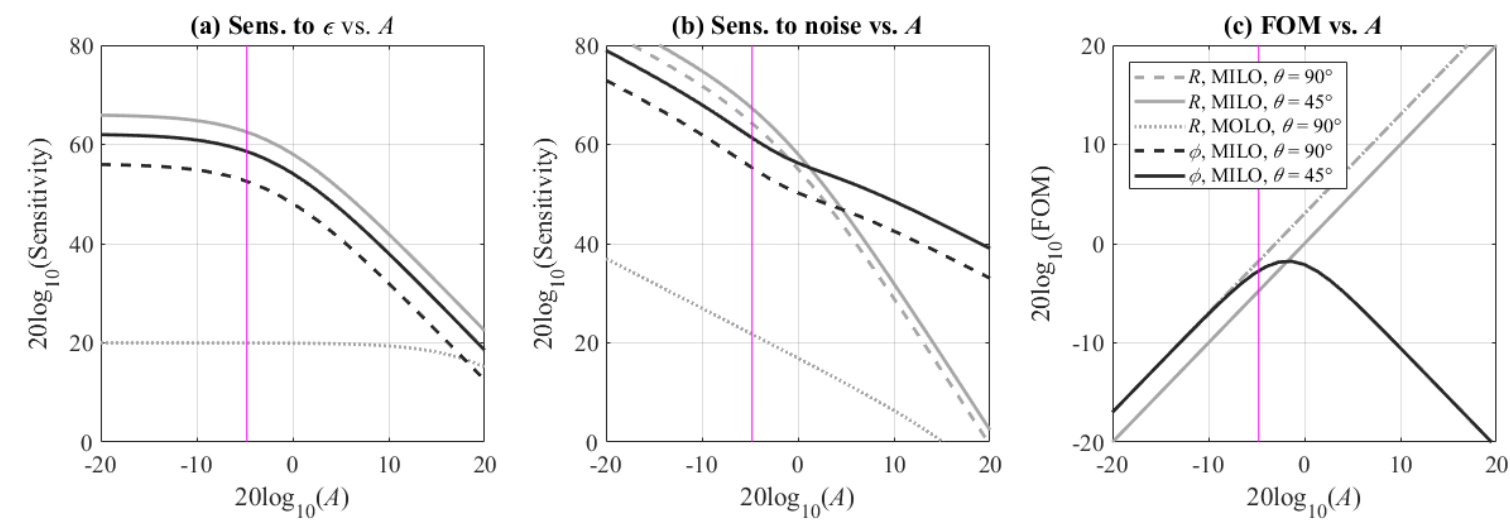

Fig. 3. Sensitivities to $\epsilon$ (a), to intrinsic noise sources (b) and FOMs (c) of different MILOs and MOLOs. Only nonlinear restoring forces are accounted for.
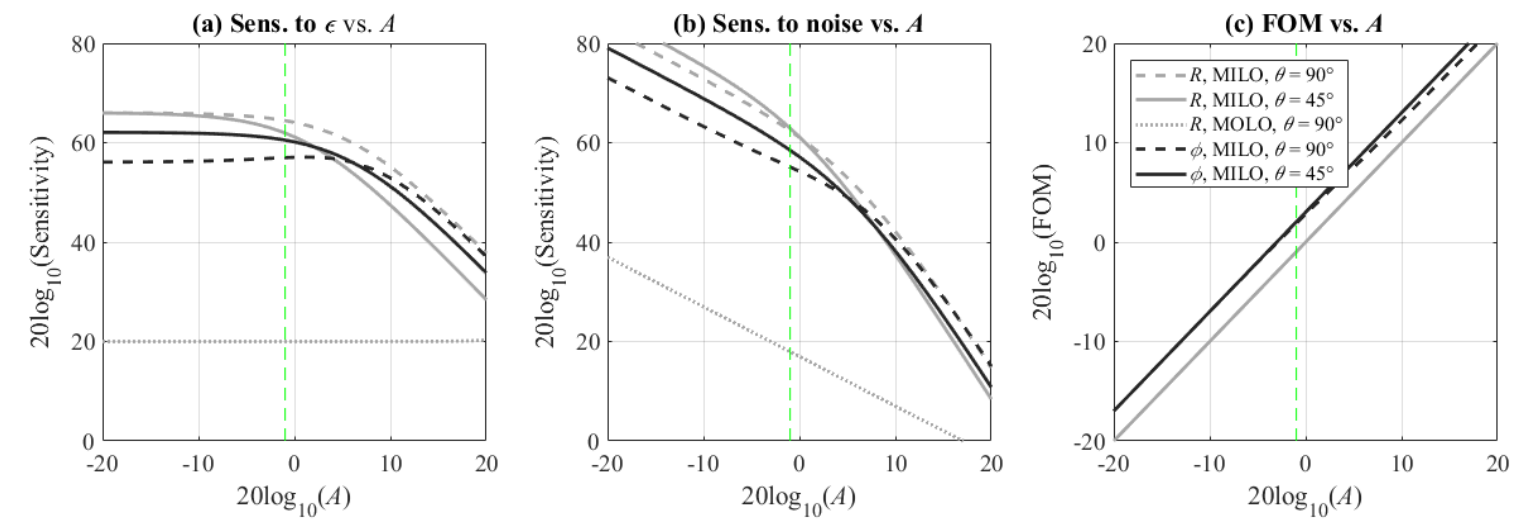

Fig. 4 - Sensitivities to $\epsilon$ (a), to intrinsic noise sources (b) and FOMs (c) of different MILOs and MOLOs. Only nonlinear damping forces are accounted for.
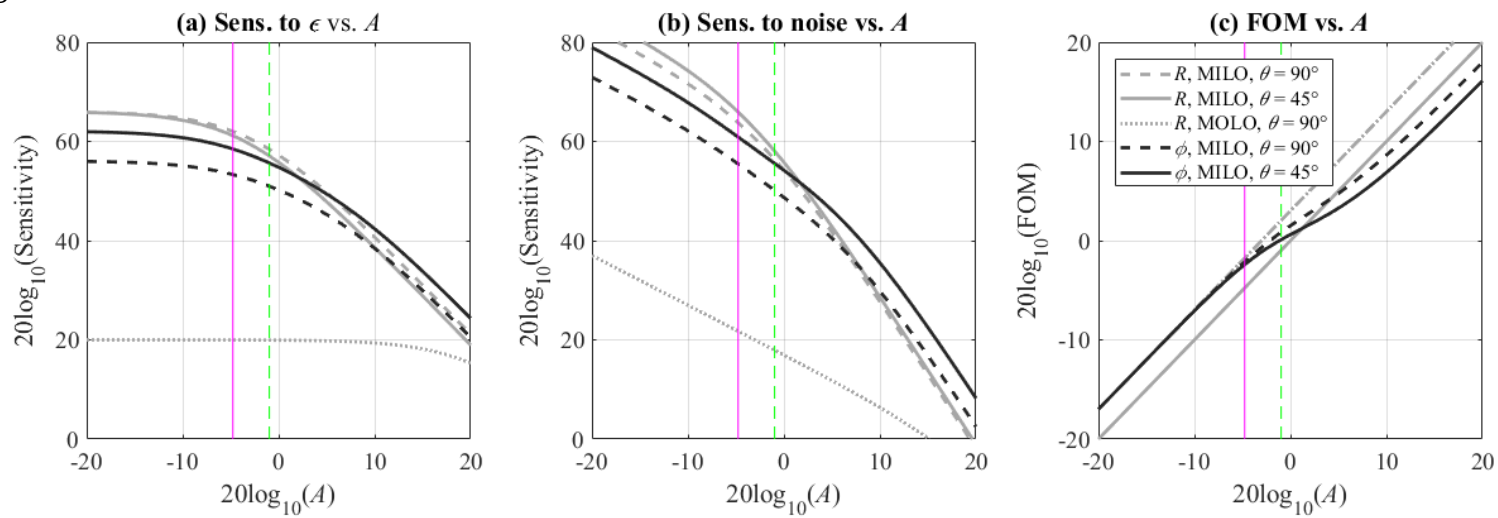

Fig. 5 - Sensitivities to $\epsilon$ (a), to intrinsic noise sources (b) and FOMs (c) of different MILOs and MOLOs. Both nonlinear restoring and damping forces are accounted for.

- consequently for all architectures, the FOMs of all output metrics increase linearly with the oscillation amplitude $A$.

In Fig. 5, both nonlinear restoring and damping forces are accounted for. With $\alpha=5 \times 10^{-4}$ and $\gamma=-2 \times 10^{-3}$, we find $A_{\text {damp }} \approx 5 \times A_{\text {Duff }}$. Most of the features of the second case are preserved except that, for $A_{\text {Duff }}<A<A_{\text {damp }}$, the FOM of $\phi$ does not increase as fast that of $R$; in fact, a decrease can even be observed when $|\gamma|$ is significantly larger than $\alpha$. Consequently, the FOM of $\phi$ is significantly smaller than that of $R$ for $A>A_{\text {damp }}$. However, the relevance of the proposed FOM may be questioned, in 
(a)

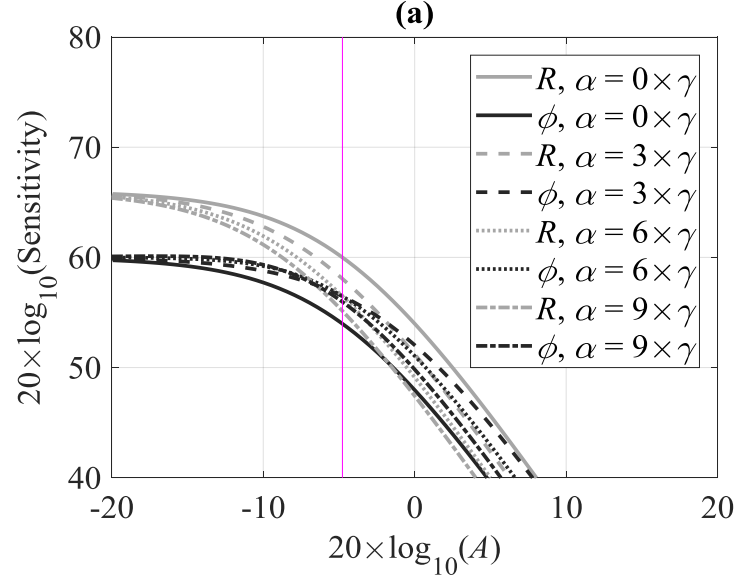

(b)

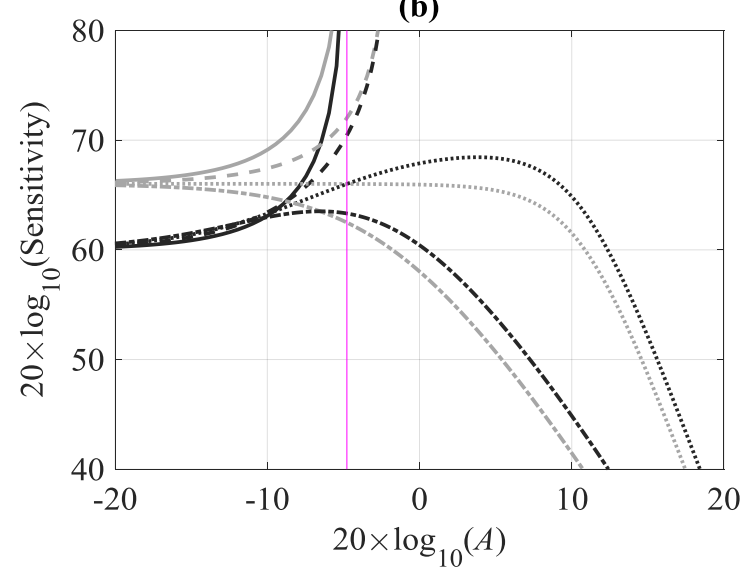

Fig. 6. Sensitivity to $\epsilon$ of amplitude ratio and phase difference, in the case $Q=10^{3}, \gamma=2 \times 10^{-3}$, and different values of $\alpha$, for the unconditionally stable mode $\phi_{0}=-90^{\circ}$ (a) and for the conditionally stable mode $\phi_{0}=90^{\circ}$ (b). The vertical line corresponds to the critical Duffing amplitude.

particular when nonlinear damping is present. This point and, more generally, the range of validity of our quasi-static analysis are addressed in the section IV.

Curves comparing the parametric sensitivities of the conditionally and unconditionally stable modes of a MILO with $\theta=90^{\circ}$ are shown in Fig. 6. The curves are drawn for $\gamma=2 \times 10^{-3}$, and

- $\alpha=0$ : only one oscillation mode $\left(\phi_{0}=-90^{\circ}\right)$ is unconditionally stable. Pure Duffing behavior.

- $\alpha=3 \gamma$ : only one oscillation mode $\left(\phi_{0}=-90^{\circ}\right)$ is unconditionally stable. Mixed nonlinear behavior.

- $\alpha=6 \gamma$ : threshold value for which the $\phi_{0}=90^{\circ}$ mode becomes unconditionally stable and nearly amplitude-independent.

- $\alpha=9 \gamma$ : both oscillation modes are unconditionally stable. With increasing $\alpha$, the two modes tend to have the same amplitude-dependence.

Qualitatively similar results are obtained when comparing the $\theta=45^{\circ}$ and $\theta=135^{\circ}$ MILO architectures.

\section{Discussion}

In this section, the dynamic properties of MILOs are illustrated: it is shown that their bandwidth is inversely commensurate with their sensitivity. We also show that the immunity of the amplitude ratio to the A-f effect reported in section III is limited to a narrow range of values of $\epsilon$, close to $\epsilon=0$, irrespective of the increase of locking range resulting from nonlinear restoring forces [9], but depending on nonlinear damping forces. Finally, practical and fundamental limits of our analysis are discussed. 


\section{V-A Dynamic properties of WCRs}

As mentioned above, the results of section III-C are valid provided the considered fluctuations are "slow". What this actually means depends on the parameters of the system and on the operating point. For the sake of brevity, we consider here only the case of a MILO with $\theta=45^{\circ}$ operating close to $\epsilon=0$, with $\gamma<0$, whose dynamic model is easily derived from (24). Using $p$ to designate the Laplace parameter, the output metric fluctuations satisfy

$$
\begin{aligned}
& \delta R=S T F_{R}(p)\left(\delta \epsilon-\frac{n_{\sin x}-n_{\sin y}}{2 A}\right)+N T F_{R}(p) \frac{n_{\cos x}-n_{\cos y}}{2 A} \\
& \delta \phi=S T F_{\phi}(p)\left(\delta \epsilon-\frac{n_{\sin x}-n_{\sin y}}{2 A}\right)+N T F_{\phi}(p) \frac{n_{\cos x}-n_{\cos y}}{2 A}
\end{aligned}
$$

where

$$
\begin{aligned}
& \operatorname{STF}_{R}(p)=\frac{1}{2}\left(\frac{1}{Q}+\frac{1}{4} \alpha A^{2}\right) \times H(p), \quad(40-\mathrm{a}) \\
& \operatorname{STF}_{\phi}(p)=-\left(p+\frac{1}{2}\left(\frac{1}{Q}+\frac{3}{4} \alpha A^{2}\right)\right) \times H(p), \\
& \operatorname{NTF}_{R}(p)=-\left(p+\frac{1}{2}\left(\frac{1}{Q}+\frac{1}{4} \alpha A^{2}\right)\right) \times H(p), \\
& \operatorname{NTF}_{\phi}(p)=\frac{3}{4 \Omega_{0}} \gamma A^{2} \times H(p),
\end{aligned}
$$

and

$$
H(p)=\frac{1}{\Omega_{0}} \frac{1}{p^{2}+\left(\frac{1}{Q}+\frac{1}{2} \alpha A^{2}\right) p+\frac{1}{2}\left(\frac{1}{Q}+\frac{1}{4} \alpha A^{2}\right)\left(\frac{1}{2}\left(\frac{1}{Q}+\frac{3}{4} \alpha A^{2}-\frac{3}{2 \Omega_{0}} \gamma A^{2}\right)\right)} .
$$

The variations of $\epsilon$ that one seeks to measure are filtered by $\operatorname{STF}_{R}(p)$, or $\operatorname{STF} F_{\phi}(p)$, the "signal transfer function", which has a second order characteristic. These variations may be considered as quasi-static within the bandwidth:

$$
\Delta \omega_{S T F}=\sqrt{\frac{1}{2}\left(\frac{1}{Q}+\frac{1}{4} \alpha A^{2}\right)\left(\frac{1}{2}\left(\frac{1}{Q}+\frac{3}{4} \alpha A^{2}\right)-\frac{3}{4 \Omega_{0}} \gamma A^{2}\right) .}
$$

which increases with $A$, provided either of the two nonlinear phenomena is present. The resonance is more or less marked depending on which is the dominant phenomenon, resulting also in more or less ringing in the sensor response. Above this bandwidth, the sensor may be considered unresponsive to $\epsilon$ - and to in-phase noise as well, since inphase noise is also filtered by $S T F_{R}(p)$ or $S T F_{\phi}(p)$. On the other hand, quadrature noise is filtered by another transfer function, $N T F_{R}(p)$ or $N T F_{\phi}(p)$, which sets another limit to the validity of the results of section III-B. From (40), these are found to be relevant only within the bandwidth: 


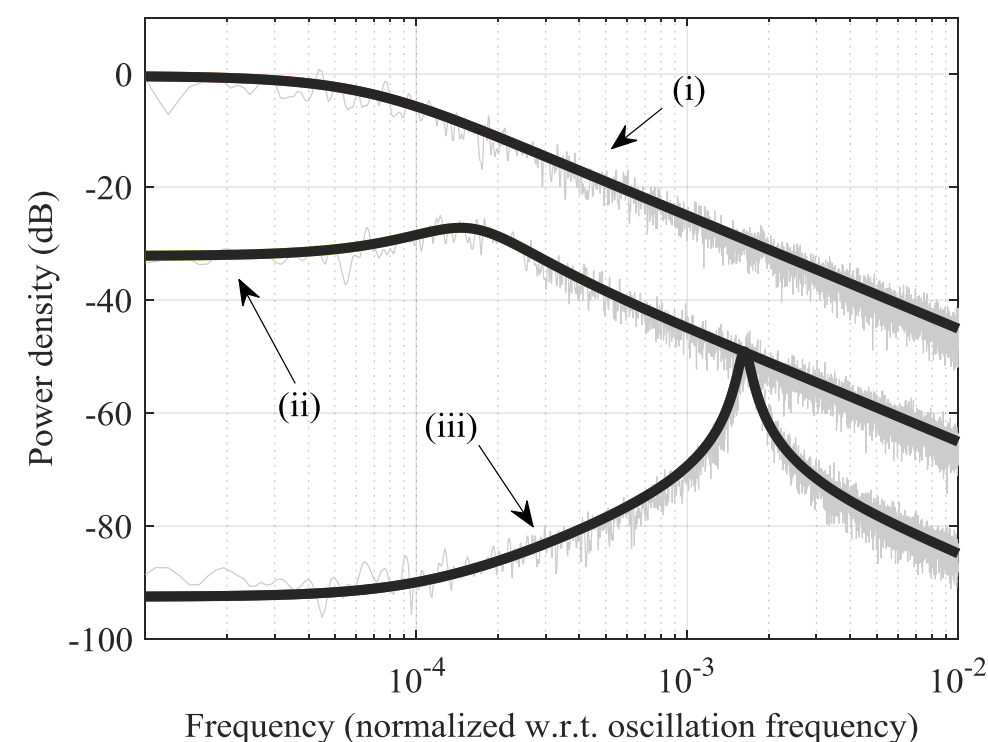

Fig. 7. Spectra of amplitude ratio fluctuations obtained for different drive levels (i) $\mathrm{F}=10^{-4}$, yielding $\Omega_{0} \approx 1$, $\mathrm{A} \approx 0.045 \approx 0.17 \times \mathrm{A}_{\text {Duff }}$, (ii) $\mathrm{F}=10^{-3}$, yielding $\Omega_{0} \approx 1, \mathrm{~A} \approx 0.45 \approx 1.7 \times \mathrm{A}_{\text {Duff }}$, (iii) $\mathrm{F}=10^{-2}, \quad$ yielding $\Omega_{0} \approx 0.90, A \approx 5.0 \approx 19.4 \times A_{\text {Duff. }}$ The thick black lines are obtained with the analytical calculations (39-41), the thin grey lines with transient simulations of (1).

$\Delta \omega_{N T F}=\frac{1}{2}\left(\frac{1}{Q}+\frac{1}{4} \alpha A^{2}\right)$

For all practical purposes, since in all stable configurations $\Delta \omega_{N T F}<\Delta \omega_{S T F}$, one may then consider as "slow" any fluctuation with a characteristic angular frequency below $\Delta \omega_{N T F}$.

These results are illustrated in Fig. 6, where the spectrum of amplitude ratio fluctuations obtained with transient simulations of (1) for different values of the drive level $F$ are compared with the analytical results derived from (39-41), in the case $\alpha=0, \gamma=$ $-10^{-2}, Q=1000$. There is an excellent match between the analytical results and the simulated ones, even at the largest drive levels.

Similar results can be derived for the other architectures studied in section III, or other WCRs, from the generic model (11).

\section{$V-B$ What $\epsilon \approx 0$ means}

In [17], the FOM of a MILO with nonlinear restoring forces and linear damping is computed for several values of $\epsilon$. These simulations show that the linear increase of the FOM of $R$ with the oscillation amplitude is only valid within a narrow range of values of $\epsilon$, commensurate with the linear locking range of the architecture, in spite of the nonlinear increase of the locking range with $A$ resulting from nonlinear restoring forces [9].

These results can be extended to the case when nonlinear damping is present in the system. In that case, the $\epsilon \approx 0$ hypothesis is found to be valid within a range of the order 
of $\Delta \omega_{N T F}$, while the nonlinear locking range is commensurate with $\Delta \omega_{S T F}$ (and may be much larger than $\Delta \omega_{N T F}$ ). This can be derived and interpreted as in section IV-A, with the impact of quadrature noise on sensor response being amplified for $|\epsilon|>\Delta \omega_{N T F}$.

This result, which also holds for the other WCRs, emphasizes that closed-loop control of mismatch (e.g. via electrostatic tuning) is a necessary feature of any practical sensing application based on coupled resonators.

\section{V-C Measurement noise}

As pointed out, the analysis and the results of section II (and those of our previous theoretical papers on similar subjects $[3,10,17])$ do not take measurement noise into account. This is because thermomechanical noise and the A-f effect are known to limit the "ultimate" performance of frequency-modulated resonant sensors [18, 27], rather than measurement noise. As we have shown, the same causes may also limit the performance of phase-modulated WCRs (Fig. 3) when nonlinear restoring forces dominate. On the other hand, in amplitude-modulated WCRs, the FOM of the amplitude ratio output metric increases linearly with $A$, no matter which nonlinearity dominates, as established in section III. Thus, intrinsic noise sources do not limit the performance of such sensors. Other error sources, such as measurement noise, should then be accounted for. To this end, a set of observer equations should be added to (1) or to (7), e.g.

$X_{\text {meas }}(t)=X(t)+N_{X}(t), Y_{\text {meas }}(t)=Y(t)+N_{Y}(t)$,

where $N_{X}$, and $N_{Y}$ represent (random) measurement errors, such as readout and quantization noise. Note that these errors may be fed back in the oscillator loop and impact the dynamics of $X, Y$ and $\phi(7)$ by contributing to $n_{x}$ and $n_{y}$ in (1). However, this is highly dependent on how the loop is implemented, in particular how the driving forces are generated (e.g. through direct feedback, as in our study, or with PLLs, with hard limiting nonlinearities or with AGCs), so that we disregard this fed-back noise and concentrate on the limit set by computing the amplitude ratio from $X_{\text {meas }}$ and $Y_{\text {meas }}$ rather than from $X$ and $Y$. Regardless of which architecture is used, we find, close to $\epsilon=0$ :

$\delta R_{\text {meas }}=\frac{A+\delta X+N_{X}}{A+\delta Y+N_{Y}}-1 \approx \frac{\partial R}{\partial \epsilon}\left(\delta \epsilon+N_{\epsilon}\right)$

where

$N_{\epsilon}=\frac{1}{A} \times\left(N_{\text {int }}+\frac{1}{\frac{\partial R}{\partial \epsilon}} N_{\text {meas }}\right)$

the sensor's input-referred noise, is the sum of two contributions: $N_{\text {meas }}=N_{X}-N_{Y}$, and $N_{\text {int }}$, which results from intrinsic noise, which is a linear combination of $n_{\cos x}, n_{\text {cosy }}$, $n_{\operatorname{sinx}}$ and $n_{\sin y}$ (e.g. (27)). Equation (46) shows that the sensitivity to mismatch and the 
oscillation amplitude should both be increased for better performance with respect to measurement noise.

Assuming $\alpha=0$, the sensitivity to mismatch of $R$ decreases as $1 / A^{2}$ above $A_{\text {Duff }}$ (Fig. 3). It follows that the contribution of measurement noise to input-referred noise (46) increases above $A_{\text {Duff }}$. Thus, it is measurement noise which ultimately limits the performance of amplitude-modulated resonant sensors based on coupled resonators ${ }^{2}$. Consequently, in practice, such sensors have an optimal operation amplitude $A_{\text {opt }}$ which minimizes input-referred noise. The value of $A_{\text {opt }}$ depends on the relative importance of intrinsic and measurement noise sources. In particular, $A_{\text {opt }}$ is greater than $A_{\text {Duff }}$ when intrinsic noise dominates.

Note also that in the threshold cases mentioned in section II-1, when $\alpha$ and $|\gamma|$ are comparable, the sensitivity to mismatch of $R$ is independent of $A$ (and that of $\phi$ slightly increases with $A$ ). Thus, in such cases, input-referred noise would simply decrease with $A$ (46).

However, the variance of thermomechanical noise becomes amplitude-dependent when $\alpha \neq 0$, as explained in sub-section $\mathrm{D}$ below, so that this reasoning must be re-examined when nonlinear damping forces dominate.

\section{V-D Nonlinear dissipation-fluctuation}

The conclusions of the above study rely on the assumption that the level of thermomechanical noise in the system is independent of $A$. However, this assumption may no longer be valid when nonlinear damping is present. While there have been many attempts to establish a proper theoretical framework for nonlinear dissipationfluctuation [32-34], no unified theory seems to emerge - to the best of our knowledge. Without pretense at exactness, one may infer the dependence of thermomechanical noise on $A$ from the linearized dynamics of the studied systems (18) (26) (34), since these equations describe the evolution of linear dissipative networks (to which linear dissipation-fluctuation applies). One may then deduce from these equations that, for $A>A_{\text {damp }}$, the variance of thermomechanical fluctuations should increase as $A^{2}$, or their standard deviation as $A$, which is coherent with the models presented in [32-34]. Consequently, the contribution of intrinsic noise sources to input-referred noise (46) will not decrease above $A_{\text {damp }}$, which sets yet another limit to what resolution may practically be reached with coupled resonators. Finally, it is worth noting that this limitation also applies to phase difference measurements.

2 This may explain the results in [13-15] for example, where the high $Q$ of the resonators leads to very little thermomechanical noise and dominant measurement noise. Conversely, the low-Q fully-integrated CMOS-MEMS MILO presented in [4] is thermomechanically-limited [12]. 


\section{Conclusion}

We have developed a complete framework of analysis for self-oscillating sensors based on two coupled resonators, e.g. MILOs and MOLOs, which may readily be extended to systems based on a larger number of resonators. Although, in this paper, we have only considered common nonlinear effects such as Duffing nonlinearity and, for the first time, quadratic damping, our analysis could also extend to other nonlinearities (e.g. actuation nonlinearity, squeezed-film damping, etc.). Based on this framework, we have proposed a general model for the sensitivity and bandwidth analysis of such systems, including intrinsic and extrinsic noise sources (11), and parametric fluctuations.

Two MILO architectures, one for which amplitude ratio $R$ is the optimal output metric $\left(\theta=90^{\circ}\right.$, studied here for the first time), the other for which phase difference $\phi$ is optimal $\left(\theta=45^{\circ}\right.$, as in [4]) were studied. Both architectures were shown to have similar properties, dependent on the relative value of the oscillation amplitude $A$ with respect to critical thresholds $A_{\text {Duff }}$ (22) and $A_{\text {damp }}$ (23). When $A_{\text {Duff }}<A_{\text {damp }}$ (as is most common), we found that, for $A>A_{\text {Duff }}$,

- at most one oscillation state is stable, depending on the hardening or softening character of the nonlinear restoring forces.

- $\quad$ the parametric sensitivity to stiffness mismatch of $R$ and $\phi$ decreases as $1 / A^{2}$.

- the sensitivity to intrinsic noise of $R$ decreases as $1 / A^{3}$, so that the FOM of $R$ increases linearly with $A$.

- the sensitivity to intrinsic noise of $\phi$ decreases as $1 / A$ if $A<A_{d a m p}$, or as $1 / A^{3}$ otherwise. In the first case, the FOM of $\phi$ decreases linearly with $A$, i.e. $\phi$ is limited by the A-f effect.

The same amplitude-dependent pattern was found to hold for a MOLO architecture operating close to the "veering zone" (in which case, $R$ is the only relevant output metric), the main difference being the exact expression of $A_{\text {Duff }}$ (37) and $A_{\text {damp }}$ (38). For the first time, we showed that, above $A_{D u f f}$, only one of the two oscillation states of such sensors is stable. As in the case of MILOs, the amplitude ratio was found to be immune to the A-f effect.

Finally, it was shown that, in MILOs, nonlinear damping could partially compensate the effect of nonlinear restoring forces when $\alpha$ and $|\gamma|$ are commensurate. In particular, we found that, provided an architecture-dependent relation between $\alpha$ and $|\gamma|$ holds, the performance of MILOs can be made amplitude-independent. Such tuning may for example be achieved by clever use of internal resonances, which is one of the avenues of research we are currently pursuing.

These properties were established in the case of nominally identical resonators $(\epsilon=0)$, which is most useful for MILOs, or for MOLOs operating in the veering zone, which is their zone of greatest sensitivity. However, it should be pointed out that the general model (11) can still be used to study other cases (e.g. MOLOs far from veering zone, with 
finite $\epsilon$ ). Other theoretical and practical limitations to these results were also investigated. We first showed that sensitivity to stiffness mismatch decreased above a certain amplitude-dependent frequency offset. This finite bandwidth limitation should always be taken into account when trying to determine sensor resolution from experimental spectra (noise above $\Delta \omega_{S T F}$ not being relevant for sensor operation). Furthermore, since sensitivity and in-band noise are both amplitude-dependent, one should also be careful to assess these two quantities in the same operating conditions, or risk overly optimistic estimations of input-referred noise, for example. We then showed that it is measurement noise which sets the practical limit to nonlinear operation of amplitude-modulated WCRs, and that there always exists an optimal oscillation amplitude $A_{\text {opt }}$ above which their input-referred noise starts increasing. Whether $A_{\text {opt }}>A_{\text {Duff }}$ depends on the relative power of intrinsic noise and measurement noise. In this respect, the development of a VLSI-compatible high-resolution analog-to-digital interface for AM WCRs presents an interesting challenge, which we hope to meet in the coming years.

It is also worth re-stating that, in the presence of nonlinear damping, quadrature and inphase intrinsic noise components should become amplitude-dependent. Our results should then be ultimately re-examined in this light, which will require further theoretical and experimental study. Barring that case, we have shown in the paper that the FOM of the amplitude ratio output metric increases linearly with $A$, while that of phase difference is limited by the A-f effect. Hence a direct gain in resolution and bias instability for nonlinear amplitude-modulated WCRs, provided thermomechanical noise is the main noise mechanism.

Of course, other noise sources may make it so that the input-referred noise is actually much larger than the thermomechanical noise floor. This is for example discussed in section IV of our paper, in which we study the impact of measurement noise on an amplitude-modulated MILO. Clearly, the contribution of measurement noise to inputreferred noise is all the weaker as the sensitivity to $\epsilon$ is larger. In that case, nonlinear operation is not desirable, since sensitivity to $\epsilon$ decreases with $A$, except in the specific case of commensurate $\alpha$ and $|\gamma|$.

This gives some general guidelines for the design of WCRs. However, these should always be re-examined in a larger, application-dependent scope. For example, the design of an analog front-end (AFE) for a resonant sensor or an oscillator is largely dependent on the expected level of the motional signals, and hence on amplitude $A$. Thus, amplitude or phase measurement noise cannot be considered independently of $A$ (as we do in section IV). Furthermore, it is unavoidable that a part of the AFE-dependent measurement noise is re-injected in the resonators through their drive signals (Leeson effect in a classical oscillator). How much depends largely on the amplitude-dependent distortion introduced by the driving and transduction schemes: in fact, electrostatic actuation nonlinearity, which is ubiquitous in MEMS resonators, may have a positive or negative impact on frequency stability [35-36], depending on $A$ and the excitation 
waveform. Other limiting phenomena, unaccounted for in the present study, are fluctuations of $\mathrm{Q}$ factor - that are bound to have a larger impact on amplitude ratio measurements than on phase difference measurements - or fluctuations of resonator bias voltage: the larger the bias voltage, the more it tends to fluctuate, leading to fluctuations of the electromechanical stiffness of the resonators and in measurement noise (as in [23]). This is yet another parameter which must be accounted for with an application in mind, and cannot be uncorrelated from the choice of an oscillation amplitude and of an AFE.

What these points show is that there is little point in saying whether nonlinear operation is good or bad out of a specific application-dependent context: oscillation amplitude $A$ is just one of the many degrees of freedom that designers have in order to optimize a resonant sensor towards a certain performance (performance may be metrological, but may also translate in terms of consumption, area, cost, etc.). For lack of proper modelling tools and of qualitative understanding of nonlinear systems, this degree of freedom is often completely neglected. We hope the present paper provides designers with such tools and understanding, and stimulates research in new directions. Deciding whether nonlinear operation is desirable or not is then highly dependent on the application, and it remains a choice which should be made on a case by case basis.

Besides the above mentioned perspectives, our ongoing work is dedicated to developing a software model for computer-aided design of WCRs, including gyroscopes (AM, FM or PM), to which the present study may also be adapted.

Finally, a detailed experimental study of the nonlinear operation of a WCR is made in [23], in which many of our theoretical results are validated.

\section{References}

[1] C. Zhao et al., "A review on coupled MEMS resonators for sensing applications utilizing mode localization", Sensors and Actuators A, vol. 249, pp. 93-111, 2016.

[2] P. Thiruvenkatanathan, J. Yan, J. Woodhouse, A. A. Seshia, "Enhancing parametric sensitivity in electrically coupled MEMS resonators", IEEE Journal of Microelectromechanical Systems, vol. 18, pp. 1077-1086, 2009.

[3] J. Juillard, P. Prache, N. Barniol, "Analysis of mutually injection-locked oscillators for differential resonant sensing", IEEE Transactions on Circuits and Systems 1, vol. 63, pp. 1055-1066, 2016.

[4] P. Prache, et al., "Design and characterization of a monolithic CMOS-MEMS mutually injection-locked oscillator for differential resonant sensing", Sensors and Actuators A, vol. 269, pp. 160-170, 2018.

[5] M. Pandit, C. Zhao, G. Sobreviela, A. A Seshia, "Immunity to Temperature Fluctuations in Weakly Coupled MEMS Resonators", 2018 IEEE Sensors Conference, pp. 1-4, 2018.

[6] G. Stemme, "Resonant silicon sensors", Journal of Micromechanics and Microengineering, vol. 1, 113, 1991. 
[7] C. Zhao et al., "A Comparative Study of Output Metrics for an MEMS Resonant Sensor Consisting of Three Weakly Coupled Resonators", IEEE/ASME Journal of Microelectromechanical Systems, vol. 25, pp. 626-636, 2016.

[8] H. Zhang, J. Yang, W. Yuan, H. Chang, "Linear sensing for mode-localized sensor", Sensors and Actuators A, vol. 277, pp.33-42, 2018.

[9] J. Juillard, A. Mostafa, P. M. Ferreira, "Nonlinear enhancement of locking range of mutually injection-locked oscillators for resonant sensing applications", European Frequency and Time Forum, pp. 109-113, 2018.

[10] J. Juillard ; P. Prache, P. M. Ferreira, N. Barniol, "Ultimate Limits of Differential Resonant MEMS Sensors Based on Two Coupled Linear Resonators", IEEE Transactions on Ultrasonics, Ferroelectrics, and Frequency Control, vol. 65, pp. 2440-2448, 2018.

[11] H.-C. Chang, X. Cuo, U.K. Mishra, R.A. York, "Phase Noise in Coupled Oscillators: Theory and Experiment", IEEE Transactions on Microwave Theory and Techniques, vol. 45, pp. 604-615, 1997.

[12] J. Juillard ; P. Prache, P. M. Ferreira, N. Barniol, "Resolution of phase difference and frequency measurements of mutually injection-locked oscillators for resonant sensing applications", Symposium on Design, Test, Integration and Packaging of MEMS and MOEMS (DTIP), pp. 1-4, 2018.

[13] M. Pandit et al., "Closed-loop Characterization of Noise and Stability in a Mode-localized Resonant MEMS Sensor", IEEE Transactions on Ultrasonics Ferroelectrics and Frequency Control, in press.

[14] M. Pandit et al., "Reduction of Amplitude Ratio Dependence on Drive Level in Mode Localized Resonant MEMS Sensors", IEEE Sensors conference, 3 pp., 2017.

[15] C. Zhao et al., "Experimental Observation of Noise Reduction in Weakly Coupled Nonlinear MEMS Resonators", IEEE/ASME Journal of Microelectromechanical Systems, vol. 26, pp. 1196-1203, 2017.

[16] O. Shoshani, S. W. Shaw, "Phase Noise Reduction and Optimal Operating Conditions for a Pair of Synchronized Oscillators", IEEE Transactions on Circuits and Systems 1, vol. 63, p. 1-11, 2016.

[17] J. Juillard, A. Mostafa, P. M. Ferreira, "Analysis of resonant sensors based on mutually injection-locked oscillators beyond the critical Duffing amplitude", European Frequency and Time Forum, pp. 114-118, 2018.

[18] E. Rubiola, "Phase noise and frequency stability in oscillators", Cambridge University Press, 2008.

[19] M.H. Bao, "Micro-mechanical transducers: pressure sensors, accelerometers and gyroscopes", Elsevier (Amsterdam), 2000.

[20] M. H. Bao, H. Yang, "Squeeze-film air damping in MEMS", Sensors and Actuators A, vol. 136, pp. 3-27, 2007.

[21] A. Gusso, "Nonlinear damping in doubly clamped beam resonators due to the attachment loss induced by the geometric nonlinearity", Journal of Sound and Vibration, vol. 372, pp. 255-265, 2016. 
[22] C. Van der Avoort et al., "Amplitude saturation of MEMS resonators explained by autoparametric resonance" Journal of Micromechanics and Microengineering, vol. 20, 105012, 2010.

[23] J. Juillard, A. Mostafa, P. M. Ferreira, "Nonlinear operation of resonant sensors based on weakly-coupled resonators: experimental validation", available as preprint on HAL, 2019.

[24] R. Lifschitz, M. C. Cross, "Nonlinear Dynamics of Nanomechanical and Micromechanical Resonators", in Review of Nonlinear Dynamics and Complexity, Wiley, 2008.

[25] J. A. Sanders, F. Verhulst, J. Murdock, "Averaging Methods in Nonlinear Dynamical Systems", in Applied Mathematical Sciences, Springer, 2000

[26] A. Gelb, W. Van der Welde, "Multiple-input describing functions and nonlinear system design", McGraw-Hill (New-York), 1968.

[27] N. Cleland, M. Roukes, "Noise processes in nanomechanical resonators", Journal of Applied Physics, vol. 92, pp. 2758-2769, 2002.

[28] Mirzaei, et al., "The quadrature LC oscillator: a complete portrait based on injection locking", IEEE Journal of Solid-State Circuits, vol. 42, pp. 1916-32, 2007.

[29] Zhao, et al., "A Closed-loop Readout Configuration for Mode-localized Resonant MEMS Sensors", IEEE/ASME Journal of Microelectromechanical Systems, vol. 26, pp. 501-503, 2017.

[30] M. Pandit, et al., "Coupled Nonlinear MEMS Resonators for Sensing”, International Frequency Control Symposium, pp. 1-4, 2018.

[31] M. Pandit et al., "Utilizing Energy Localization in Weakly Coupled Nonlinear Resonators for Sensing Applications", IEEE Journal of Microelectromechanical Systems, vol. 28, pp. 185-188, 2019.

[32] N. G. Van Kampen, "Thermal fluctuations in nonlinear systems", Journal of Mathematical Physics, vol; 4, pp. 190-194, 1963.

[33] G. J. Coram, B. D. O. Anderson, J. L. Wyatt Jr., "Limits to the FluctuationDissipation Theorem for Nonlinear Circuits", IEEE Transactions on Circuits and Systems 1, vol. 47, pp. 1323-1329, 2000.

[34] L. Weiss, W. Mathis, "A thermodynamical approach to noise in nonlinear networks", International Journal of Circuit Theory and Applications, vol. 26, pp. 147-165, 1998.

[35] J. Juillard, A. Brenes, "Impact of excitation waveform on the frequency stability of electrostatically-actuated micro-electromechanical oscillators", Journal of Sound and Vibration, vol. 422, pp. 79-91, 2018.

[36] J. Juillard, A. Brenes, M. Gouspy, M. Kraft, "Towards the optimization of excitation waveform for electrostatic resonant sensors with enhanced frequency stability", Symposium on Design, Test, Integration and Packaging of MEMS/MOEMS, DTIP 2019, 4 pp. 